\title{
COMPOSIÇÃO FÍSICO-QUÍMICA E QUALIDADE DO CAFÉ SUBMETIDO A DOIS TIPOS DE TORRAÇÃO E COM DIFERENTES FORMAS DE PROCESSAMENTO
}

\author{
Physical-chemical composition and quality of coffee submitted to two roasting procedures and to \\ different methods of preparation
}

\author{
Heloisa Helena de Siqueira ${ }^{1}$, Celeste Maria Patto de Abreu ${ }^{2}$
}

\begin{abstract}
RESUMO
Este estudo foi desenvolvido com o objetivo de determinar as alterações na composição físico-química, química e sensorial de café cultivar Rubi, o qual foi submetido a diferentes formas de processamento e dois tipos de torração. Foram colhidos cafés de uma lavoura da UFLA com experimento de pivô central. Após o beneficiamento, as amostras foram divididas em cafés crus e torrados, sendo realizadas as seguintes análises: $\mathrm{pH}$, acidez titulável total, cafeína, ácido clorogênico, polifenóis e índice de coloração. Foi realizada também análise sensorial (prova de xícara) para determinação da qualidade da bebida. Para a variável polifenol, não houve diferença significativa entre as formas de processamento, sendo que a torração clara apresentou maior teor de polifenol. O café natural apresentou um maior valor de cafeína dentro do tipo de processamento, e dentro do tipo de grão, o grão cru apresentou um maior valor de cafeína. Para a variável índice de coloração, os processamentos despolpado e descascado apresentaram os maiores valores e a torração média também apresentou um maior valor. O processamento natural apresentou um maior teor de ácido clorogênico e para o tipo de grão, a torração média também apresentou um maior valor de ácido clorogênico. Para a variável pH, não houve diferença significativa entre as formas de processamento, e dentro do tipo de grão, o grão cru apresentou um maior valor de $\mathrm{pH}$. Com relação à acidez, o café natural apresentou um maior valor desta variável, e a torração média também apresentou um maior valor. Não houve diferença entre as formas de processamento e tipo de torração em relação à análise sensorial, visto que, todos os cafés foram classificados como bebida dura.
\end{abstract}

Termos para indexação: Processamento, torração, qualidade.

\begin{abstract}
This study was carried out to determine alterations in the physical-chemical, chemical and sensorial composition of a Rubi cultivar coffee, which was submitted to different methods of preparation and two roasting procedures. The coffees were harvested in a farm at UFLA with a central pivot design. After processing, samples were divided into raw and roasted coffees and the following parameters were evaluated: $\mathrm{pH}$, total titratable acidity, caffeine, chlorogenic acid, polyphenols and color index. Sensorial analysis up test was also performed to determine beverage quality. For the variable polyphenol, there were no significant differences among the methods of preparation, though the light roast coffee showed the greatest polyphenol content. The natural coffee presented the greatest amount caffeine within the type of preparation, while the raw grain achieved the highest caffeine content when all type of grains were compared. The pulped and peeled preparations, as well as the medium roast. The greatest amount of chlorogenic acid was obtained with the natural preparation or when medium roast procedure was performed. For the variable $\mathrm{pH}$, there were no significant differences among the methods of preparation, while from all types of grain, the raw one showed the highest $\mathrm{pH}$ value. Regarding total titratable acidity, the natural coffee showed the greatest value for such a variable, as well as the medium roast coffee. There was no difference among the methods of preparation and of roasting procedure during the sensorial analysis and all coffees were classified as hard beverage.
\end{abstract}

Index terms: Preparation, roasting, quality.

(Recebido para publicação em 20 de dezembro de 2003 e aprovado em 7 de julho de 2005)

\section{INTRODUÇÃO}

O café é um dos produtos agrícolas cujo processamento requer especial atenção, a fim de manter preservadas as suas qualidades. Devido a isso, o café pode ser processado de duas maneiras: via seca, que produz o café em coco; e via úmida, que produz café despolpado e descascado (IBC, 1985).
A torração é considerada uma das etapas mais importantes para o desenvolvimento do sabor e aroma do café, que são conferidos por compostos voláteis, presentes nos grãos antes e, ou, após a torração. Nesta fase, os grãos sofrem algumas reações químicas importantes, necessárias à formação da qualidade sensorial, assim como ocorrem mudanças físicas que podem variar de uma espécie a outra e também entre cultivares (LOPES, 2000).

\footnotetext{
${ }^{1}$ Doutoranda em Ciência dos Alimentos no Departamento de Ciências dos Alimentos da Universidade Federal de Lavras/UFLA - Cx. P. 3037 - $37200-000$ Lavras, MG - hh.siqueira@zipmail.com.br

${ }^{2}$ Professora Adjunta do Departamento de Química da Universidade Federal de Lavras/UFLA - Cx. P. 3037 - $37200-000$ - Lavras, MG.
} 
A acidez percebida no café é um atributo importante para análise sensorial do produto, sabendo que sua intensidade varia em função do estádio de maturação dos frutos, local de origem, tipo de colheita, forma de processamento, tipo de secagem e condições climáticas durante a colheita e secagem.

As variações de $\mathrm{pH}$ com a torração, segundo Sivetz \& Desrosier (1979), podem ser de muita importância na aceitação do produto pelo consumidor. Os mesmos autores indicam que o pH ideal deve estar entre 4,95 a 5,20 tornando o café palatável, sem excesso de amargor ou acidez.

Sabe-se que o $\mathrm{pH}$ é indicativo de eventuais transformações dos frutos de café, como as fermentações indesejáveis que ocorrem na pré ou pós-colheita, originando defeitos.

A cafeína ocorre livre no citoplasma, complexada com o clorogenato de potássio, o qual é pouco solúvel encontrando nesta forma alguma mobilidade entre os tecidos (BAUMMAM et al., 1993, citado por ILLY \& VIANNI, 1995). Conforme este mesmo autor, a cafeína não tem efeito na qualidade sensorial, sendo bastante estável com a torração.

Cerca de 32-52\% dos ácidos clorogênicos são degradados durante a torração e os produtos formados são encontrados no aroma do café (CLIFFORD, 1999). Segundo Dentam (1985), os ácidos clorogênicos ocorrem na superfície do grão associados com a graxa cuticular e, também, no citoplasma. Ainda não se sabe se a composição varia com a posição no grão.

O teor de polifenóis livres é pequeno no café verde, aumentando durante a torração desse grão. Este aumento, segundo Trugo \& Macrae (1989), está relacionado à degradação dos ácidos clorogênicos. Com a torração, os polifenóis contribuem de maneira significativa para o aroma e sabor do produto final, sendo considerados responsáveis pela adstringência dos frutos (RAMIREZ, 1987).

As evidências de que a cor dos grãos de café correlaciona-se com a qualidade do produto são enfatizadas por diversos autores (AMORIM et al., 1977; LOPES et al., 1998; NORTHMORE, 1968). Esses pesquisadores relatam as diversas cores que os grãos de café podem assumir e citam a tonalidade azul para verde como indicativa de grãos de melhor qualidade, mediante a prova de xícara.

Diante deste contexto, este trabalho foi desenvolvido visando analisar os tipos de processamento, a irrigação e os tipos de torração com relação à qualidade e as alterações na composição físico-química, química e sensorial do café.

\section{MATERIAL E MÉTODOS}

Foram colhidos cafés (Coffea arabica L.), cultivar Rubi, por derriça manual no pano, em lavouras da UFLA, oriundos de um experimento com pivô central. Os cafés foram submetidos a diferentes tipos de processamentos: cereja natural, cereja despolpado e cereja descascado, os quais foram secos em terreiros de alvenaria. As amostras foram divididas em café cru e café torrado, sendo esta torração clara e média. Foram avaliadas: acidez titulável total, pH, cafeína, ácido clorogênico, polifenol e índice de cor. As análises foram feitas no Departamento de Ciência dos alimentos e no Laboratório de Qualidade do Café "Dr. Alcides Carvalho"/ EPAMIG em Lavras, MG.

Foi realizada também avaliação sensorial (prova de xícara) no Pólo Tecnológico e Laboratório de Qualidade do Café da Universidade Federal de Lavras.

A acidez titulável foi determinada segundo o método da AOAC (1990) e a partir do mesmo extrato o pH foi medido utilizando o peagâmetro marca DIGIMED. A cafeína foi avaliada segundo o método colorimétrico descrito pelo Instituto Adolfo Lutz (1985). O ácido clorogênico também foi avaliado pelo método do ácido clorogênico total do Instituto Adolfo Lutz (1985). O índice de coloração foi determinado pelo método descrito por Singleton (1966) adaptado para o café. Os polifenóis foram extraídos pelo método de Goldstein \& Swan (1963) e determinado pelo método de Folin Denis, descrito pela AOAC (1990).

O delineamento experimental utilizado foi o inteiramente casualizado com quatro repetições, com o fatorial (3 x 3). Foi utilizado o teste Tukey a $1 \%$ de probabilidade.

\section{RESULTADOS E DISCUSSÃO}

Os teores médios de acidez titulável total e $\mathrm{pH}$ em função dos diferentes tipos de processamento e tipos de torração são apresentados na Tabela 1.

Não se observaram diferenças significativas entre os tipos de processamentos em relação aos valores de $\mathrm{pH}$. Para o tipo de grão, os valores de $\mathrm{pH}$ apresentaram-se maiores no grão cru e menores na torração clara. O café natural apresentou um maior valor tanto no grão cru quanto na torração média, o mesmo não ocorrendo com os demais.

Os valores de $\mathrm{pH}$ encontrados pela OIC (1992) estão na faixa de 5,31 a 5,61 para amostras de cafés comerciáveis. No presente trabalho, os valores de $\mathrm{pH}$ com a torração média estão dentro da faixa proposta pela OIC (1992). Os valores do grão cru $(5,73$ a 5,88$)$ encontrados nesse trabalho estão de acordo com o trabalho de Barrios (2001). 
Segundo Sivetz \& Desrosier (1979), as variações de pH com a torração pode ser de suma importância na aceitação do produto pelo consumidor e indicam que o $\mathrm{pH}$ ideal é de 4,95 a 5,20 para tornar o café palatável. Os resultados de $\mathrm{pH}$ do presente trabalho, quanto à torração média superou o $\mathrm{pH}$ ideal, podendo este café apresentarse com ligeiro excesso de amargor ou acidez.

Dentro do tipo de processamento, o café natural apresentou um maior valor de acidez quando comparado com os demais, que não diferiram entre si. O café natural manteve o mesmo comportamento em relação ao tipo de grão, apresentando os maiores valores de acidez.O café despolpado obteve os maiores valores de acidez no grão cru e na torração média. E o café descascado somente apresentou o maior valor na torração média.
Com a torração média os valores de acidez não diferiram entre os diferentes tipos de processamento.

Segundo Carvalho et al. (1994), a acidez dos grãos de café beneficiado tem relação inversa com a qualidade do café. A acidez tem um valor mais alto para o café torrado que no café verde. Estes mesmos autores detectaram maior acidez em cafés de pior qualidade.

Os cafés natural e despolpado apresentaram os maiores valores de cafeína dentro do grão cru, não diferindo significativamente entre si. Na torração clara e média, o café natural também apresentou os maiores valores. À medida que aumentava o grau de torração, os valores de cafeína apresentavam-se com um ligeiro decréscimo. Dentro do tipo de grão, o grão cru apresentou o maior valor e a torração média o menor valor.

TABELA 1 - Valores médios de pH de um café irrigado com diferentes tipos de processamento.

\begin{tabular}{|c|c|c|c|c|c|}
\hline \multicolumn{6}{|c|}{ Grão } \\
\hline Processamento & Cru & T. clara & T.média & Total & Total \\
\hline Natural & $5,88 \mathrm{Aa}$ & $5,10 \mathrm{Bc}$ & $5,50 \mathrm{Ab}$ & $5,49 \mathrm{~A}$ & \\
\hline Despolpado & $5,77 \mathrm{Ba}$ & $5,15 \mathrm{Bc}$ & $5,46 \mathrm{Ab}$ & $5,46 \mathrm{~A}$ & 5,46 \\
\hline Descascado & $5,73 \mathrm{Ba}$ & $5,27 \mathrm{Ab}$ & $5,34 \mathrm{Bb}$ & $5,45 \mathrm{~A}$ & \\
\hline Total & $5,79 \mathrm{a}$ & $5,17 \mathrm{c}$ & $5,43 \mathrm{~b}$ & & \\
\hline
\end{tabular}

*Médias seguidas pela mesma letra maiúscula na coluna e pela mesma letra minúscula na linha, não diferem entre si, a $5 \%$ de probabilidade, pelo teste Tukey.

TABELA 2 - Valores médios de acidez titulável total (NaOH.100g a 0,1N) de um café irrigado com diferentes tipos de processamento.

\begin{tabular}{|c|c|c|c|c|c|}
\hline \multicolumn{6}{|c|}{ Grão } \\
\hline Processamento & $\mathrm{Cru}$ & T. clara & T.média & Total & Total \\
\hline Natural & $250,37 \mathrm{Ab}$ & $400,30 \mathrm{Aa}$ & $400,37 \mathrm{Aa}$ & $350,35 \mathrm{~A}$ & \\
\hline Despolpado & $250,15 \mathrm{ABc}$ & $350,15 \mathrm{Bb}$ & $400,32 \mathrm{Aa}$ & $333,54 \mathrm{~B}$ & 339,11 \\
\hline Descascado & $250,07 \mathrm{Bc}$ & $350,12 \mathrm{Bb}$ & $400,12 \mathrm{Aa}$ & $333,44 \mathrm{~B}$ & \\
\hline Total & $250,20 \mathrm{c}$ & $366,86 \mathrm{~b}$ & $400,27 \mathrm{a}$ & & \\
\hline
\end{tabular}

*médias seguidas pela mesma letra maiúscula na coluna e pela mesma letra minúscula na linha, não diferem entre si, a $5 \%$ de probabilidade, pelo teste Tukey.

TABELA 3 - Valores médios de cafeína (\%) de um café irrigado com diferentes tipos de processamento.

\begin{tabular}{|c|c|c|c|c|c|}
\hline \multicolumn{6}{|c|}{ Grão } \\
\hline Processamento & Cru & T. clara & T.média & Total & Total \\
\hline Natural & $0,96 \mathrm{Aa}$ & $0,86 \mathrm{Ab}$ & $0,84 \mathrm{Ab}$ & $0,89 \mathrm{~A}$ & \\
\hline Despolpado & $0,97 \mathrm{Aa}$ & $0,79 \mathrm{Bb}$ & $0,69 \mathrm{Cc}$ & $0,81 \mathrm{~B}$ & 0,83 \\
\hline Descascado & $0,87 \mathrm{Ba}$ & $0,76 \mathrm{Cb}$ & $0,72 \mathrm{Bc}$ & $0,79 \mathrm{C}$ & \\
\hline Total & $0,94 \mathrm{a}$ & $0,80 \mathrm{~b}$ & $0,74 \mathrm{c}$ & & \\
\hline
\end{tabular}

*médias seguidas pela mesma letra maiúscula na coluna e pela mesma letra minúscula na linha, não diferem entre si, a $5 \%$ de probabilidade, pelo teste Tukey.

Ciênc. agrotec., Lavras, v. 30, n. 1, p. 112-117, jan./fev., 2006 
Este composto permanece quase inalterado na torração, com exceção de frações mínimas que são sublimadas a $176^{\circ} \mathrm{C}$ e que se acumulam nas pilhas de cafés torrados. Por outro lado, Fobé et al. (1967/1968) encontraram variações nos teores de cafeína entre diferentes graus de torração.

$\mathrm{O}$ valor do ácido clorogênico variou entre os três tipos de processamento, sendo o café descascado o que apresentou maior valor para o grão cru. Com a torração, os valores do ácido clorogênico apresentaram um ligeiro aumento, sendo a torração média a que apresentou o maior valor. Os diferentes tipos de processamentos seguiram o mesmo comportamento com a torração, no qual o café natural apresentou o maior valor e o café descascado o menor valor.

Carelli et al. (1974) verificaram teores de ácido clorogênico para a espécie Coffea arábica variando entre 6,63 a 8,20\%, e para a espécie Coffea canephora de 10,3\%. Os resultados do presente trabalho estão abaixo do citado por este autor.

Para o índice de cor, o café despolpado apresentou o maior valor desta variável para o café cru. Entretanto, na torração clara e média, o café natural apresentou os maiores índices de coloração. O mesmo comportamento pode ser observado nos três tipos de processamento com relação à torração, em que o café natural apresentou o maior valor e o café despolpado o menor valor no índice de coloração.

Os resultados do índice de cor estão de acordo com
Carvalho et al. (1989), que constataram um maior índice para os cafés de melhor qualidade e que os cafés de piores bebidas apresentaram índices de coloração inferiores a 0,70 , o que pode ser observado para o café natural dentro do grão cru.

A cor é o principal controlador do ponto final de torração. Na maioria das indústrias, através de uma amostra padrão, controla-se continuamente a cor do café que está sendo torrado (ILLY \& VIANNI, 1995). Para o café cru, a cor é a característica que chama a atenção na comercialização (AMORIM et al., 1976; MÔNACO, 1961).

Os valores de polifenóis não diferiram significativamente entre os três tipos de processamentos. Para o grão cru, esses valores também não diferiram entre os processamentos. Com a torração clara os valores de polifenóis aumentaram, sendo que o café natural e despolpado não diferiram entre si, obtendo os maiores valores. Na torração média esses valores decresceram, e o café natural apresentou o maior valor de polifenol.

A concentração de polifenóis é inversamente proporcional à qualidade da bebida, sendo diretamente relacionada ao grau de ataque de microorganismos e ao estádio de maturação dos frutos (CARVALHO et al., 1989).

Em relação à análise sensorial, verificou-se que não houve diferença na qualidade do café produzido com irrigação, em função de diferentes tipos de processamento e dos diferentes graus de torração, sendo os cafés classificados como padrão de bebida dura.

TABELA 4 - Valores médios de ácido clorogênico (\%) de um café irrigado com diferentes tipos de processamento.

\begin{tabular}{|c|c|c|c|c|c|}
\hline \multicolumn{6}{|c|}{ Grão } \\
\hline Processamento & Cru & T. clara & T.média & Total & Total \\
\hline Natural & $3,81 \mathrm{Bc}$ & $5,77 \mathrm{Ab}$ & 6,75 Aa & 5,44 A & \\
\hline Despolpado & $2,77 \mathrm{Cc}$ & $4,01 \mathrm{Bb}$ & $5,76 \mathrm{Ba}$ & $4,18 \mathrm{C}$ & 4,72 \\
\hline Descascado & 4,94 Aa & $3,86 \mathrm{Cc}$ & $4,81 \mathrm{Cb}$ & $4,54 \mathrm{~B}$ & \\
\hline Total & $3,84 \mathrm{c}$ & $4,55 \mathrm{~b}$ & $5,77 \mathrm{a}$ & & \\
\hline
\end{tabular}

*Médias seguidas pela mesma letra maiúscula na coluna e pela mesma letra minúscula na linha, não diferem entre si, a $5 \%$ de probabilidade, pelo teste Tukey.

TABELA 5 - Valores médios de índice de coloração de um café irrigado com diferentes tipos de processamento.

\begin{tabular}{lccccc}
\hline & \multicolumn{3}{c}{ Grão } & & \\
\hline Processamento & Cru & T. clara & T.média & Total & Total \\
\hline Natural & $0,69 \mathrm{Cc}$ & $1,13 \mathrm{Ab}$ & $2,00 \mathrm{Aa}$ & $1,28 \mathrm{~B}$ & \\
Despolpado & $1,44 \mathrm{Ab}$ & $0,84 \mathrm{Cc}$ & $1,78 \mathrm{Ca}$ & $1,35 \mathrm{~A}$ & 1,32 \\
Descascado & $1,10 \mathrm{Bb}$ & $0,94 \mathrm{Bc}$ & $1,96 \mathrm{Ba}$ & $1,33 \mathrm{~A}$ & \\
Total & $1,08 \mathrm{~b}$ & $0,97 \mathrm{c}$ & $1,92 \mathrm{a}$ & & \\
\hline
\end{tabular}

*médias seguidas pela mesma letra maiúscula na coluna e pela mesma letra minúscula na linha, não diferem entre si, a $5 \%$ de probabilidade, pelo teste Tukey. 
TABELA 6 - Valores médios de polifenóis (\%) de um café irrigado com diferentes tipos de processamento.

\begin{tabular}{|c|c|c|c|c|c|}
\hline \multicolumn{6}{|c|}{ Grão } \\
\hline Processamento & Cru & T. clara & T.média & Total & Total \\
\hline Natural & $6,49 \mathrm{Ac}$ & $9,22 \mathrm{Aa}$ & $7,80 \mathrm{ABb}$ & $7,84 \mathrm{~A}$ & \\
\hline Despolpado & $6,25 \mathrm{Ac}$ & $9,00 \mathrm{Aa}$ & 7,55 Bb & $7,60 \mathrm{~A}$ & 7,59 \\
\hline Descascado & $6,05 \mathrm{Ac}$ & $8,37 \mathrm{Ba}$ & 7,60 Bb & $7,34 \mathrm{~A}$ & \\
\hline Total & $6,26 \mathrm{c}$ & $8,86 \mathrm{a}$ & $7,65 \mathrm{~b}$ & & \\
\hline
\end{tabular}

*médias seguidas pela mesma letra maiúscula na coluna e pela mesma letra minúscula na linha, não diferem entre si, a $5 \%$ de probabilidade, pelo teste Tukey.

TABELA 7 - Resultados da análise sensorial de um café irrigado com diferentes tipos de processamento.

\begin{tabular}{ccc}
\hline Tipo de Café & Torração & Bebida \\
\hline Natural & Clara & Dura \\
Despolpado & Clara & Dura \\
Descascado & Clara & Dura \\
Natural & Média & Dura \\
Despolpado & Média & Dura \\
Descascado & Média & Dura \\
\hline
\end{tabular}

\section{CONCLUSÕES}

Independente do sistema de processamento e tipos de torração, os cafés apresentaram boa qualidade, sendo classificados como bebida dura.

Para o tipo de grão, torração clara e torração média, o café natural apresentou os melhores resultados nas variáveis: $\mathrm{pH}$, acidez, cafeína e polifenóis. Somente na variável $\mathrm{pH}$ com torração clara, o café natural apresentou o menor valor.

O café despolpado, no tipo de grão, apresentou melhores resultados juntamente com o café natural em: polifenóis, cafeína, acidez, diferindo deste em índice de coloração, na torração clara apresentou o melhor resultado em polifenóis e na torração média em acidez e pH.

Para tipo de grão, o café descascado somente obteve o maior valor na variável ácido clorogênico. Não variando nos teores de polifenóis com o café natural e despolpado. Na torração clara, o café descascado apresentou maior valor de $\mathrm{pH}$ e na torração média também não houve variação dos três tipos de processamento quanto aos valores de acidez.

\section{REFERÊNCIAS BIBLIOGRÁFICAS}

AMORIM, H. V.; CRUZ, A. R. M.; DIAS, R. M.; GUTIERREZ, L. E.; OLIVEIRA, G. D.; MELO, M.; TEIXEIRA, A. A. Transformações químicas e estruturais durante a deterioração da qualidade do café. In: CONGRESSO BRASILEIRO DE PESQUISAS CAFEEIRAS, 5., 1977, Guarapari. Resumos... Rio de Janeiro: IBC/GERCA, 1977. p. 15-18.
AMORIM, H. V.; LEGENDRE, M. G.; AMORIM, V. L.; ANGELO, A. J. S.; ORY, R. L. Chemistry of Brazilian green coffee and the quality of the beverage: VII. total carbanyls, activity of polyphenol oxidase, and hydroperoxides. Turrialba, San José, v. 26, n. 2, p. 193-195, 1976.

ASSOCIATION OF OFFICIAL ANALYTICAL CHEMISTS. Official methods of analyses of the Association of Official Analytical Chemists. 15. ed. Washington, 1990. $684 \mathrm{p}$.

BARRIOS, B. B. E. Caracterização física, química, microbiológica e sensorial de cafés (Coffea arábica $\mathrm{L}$.) da região Alto Rio Grande-Sul de Minas Gerais. 2001. 72 p. Dissertação (Mestrado em Ciência dos Alimentos) Universidade Federal de Lavras, Lavras, 2001.

CARELLI, M. L. C.; LOPES, C. R.; MONACO, L. C. Chlorogenic acid content in species of Coffea and selections of Coffea Arabica. Turrialba, San José, v. 24, n. 4, p. 398-401, 1974.

CARVALHO, V. D. de; CHALFOUN, S. M.; CHAGAS, S. J. de R. Relação entre classificação do café pela bebida e composição físico-química, química e microflora do grão beneficiado. In: CONGRESSO BRASILEIRO DE PESQUISAS CAFEEIRAS, 15., 1989, Maringá, PR. Anais... Rio de Janeiro: MEC/IBC, 1989. p. 25-26. 
CARVALHO, V. D. de; CHALFOUN, S. M.; CHAGAS, S. J. de R.; BOTREL, N.; JUSTE JÚNIOR, E. S. G. Relações entre a composição físico-química e química do grão beneficiado e da qualidade de bebida do café. Pesquisa Agropecuária Brasileira, Brasília, v. 29, n. 3, p. 449-454, mar. 1994.

CLIFFORD, M. N. Chlorogenic acids and other cinnamates nature, occurrence and dietary burden. Journal of the Science Food and Agriculture, Oxford, v. 79, p. 363-372, 1999.

DENTAM, E. The microscopic structure of the coffee bean. In: . Botany, biochemistry and production of bean and beverages. London: Croon Helm, 1985.

FOBÉ, L. A.; NERY, J. P.; TANGO, J. S. Influência do grau de torração sobre a composição química do café. Coletânea do Instituto de Tecnologia de Alimentos, Campinas, v. 2, p. 251-268, 1967/1968.

GOLDSTEIN, J. L.; SWAN, T. Changes in tannins in ripening fruits. Phytochemistry, Oxford, v. 2, p. 371-382, 1963.

ILLY, A.; VIANNI, R. Expresso coffee: the chemistry of quality. San Diego: Academic, 1995. 235 p.

INSTITUTO ADOLFO LUTZ. Normas analíticas, métodos químicos e físicos para análise de alimentos. 3. ed. São Paulo, 1985. v. 1.

INSTITUTO BRASILEIRO DO CAFÉ. Cultura do café no Brasil: manual de recomendações. 5. ed. Rio de Janeiro: IBC-GERCA, $1985.580 \mathrm{p}$.

LOPES, L. M. V. Avaliação da qualidade de grãos crus e torrados de cultivares de cafeeiro (Coffea arabica $L$.). 2000. 95 p. Dissertação (Mestrado em Ciência dos Alimentos) - Universidade Federal de Lavras, Lavras, 2000.
LOPES, R. P.; HARA, T.; SILVA, J. S. Avaliação da qualidade de grãos de café pela colorimetria. Engenharia na Agricultura, Viçosa, v. 6, n. 3, p. 160-169, 1998.

MÔNACO, L. C. Armazenamento de café. Boletim da Superintendência dos Serviços do Café, São Paulo, v. 36, n. 417, p. 15-16, 1961.

NORTHMORE, J. M. Raw bean colors and the quality of Kenya Arabica coffee. Turrialba, San José, v. 18, n. 1, p. 14-20, 1968.

ORGANIZACIÓN INTERNACIONAL DEL CAFÉ. El despulpado del café por medio de desmucilaginadoras mecanicas sin proceso de fermentación y su efecto en la calidade de bebida de café producido en la región de Apucaran en el estado de Paraná en Brasil. [S.1.], 1992. Não paginado. (Reporte de Evolución Sensorial).

RAMIREZ, J. Compuestos fenólicos en la pulpa de café: cromatografía de papel de pulpa fresca de 12 cultivares de Coffea arabica L. Turrialba, San José, v. 37, n. 4, p. 317323, oct./dic. 1987.

SINGLETON, V. L. The total pheneolic content of grape berries during the maturation of several varieties. American Journal of Enology and Viticulture, Davis, v. 17, p. 126134, 1966.

SIVETZ, M.; DESROSIER, N. W. Physical and chemical aspects of coffee. Coffee Techonology, Westpor, p. 527575, 1979.

TRUGO, L. C.; MACRAE, R. Application of high performance liquid chromatography to the analysis of some non volatile coffee components. Archivos Latinoamericanos de Nutricion, Caracas, v. 39, n. 1, p. 96107, Mar. 1989. 